العدد الثاني عشر - الجزء الثاني

مجلة العمارة والقنون

تأثير التطور التكنولوجى للكاميرات على الشكل التهائى للصورة المتحركة فى المحتوى المرئى

The Impact of Technological Development of the Cameras on the Final Form of the Motion Picture in Visual Content

أ.د/ خالا على عويس أليس

عميد المعهد العالى للفنون التطبيقية ــ أكاديمية القاهرة الجديدة للعلوم والفنون بالتجمع الخامس

Prof. Dr. Khaled Ali Awes

Dean of the Higher Institute of Applied Arts - New Cairo Academy of Sciences and Arts at the 5th Settlement

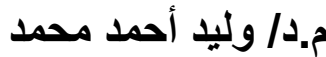

مدرس بقسم الفوتوغرافيا والسينما والتلفزيون - كلية الفنون التطبيقية ـ جامعة حلوان

Assist. Dr. Walid Ahmed Mohamed

Lecturer at the Department of Photography, Cinema and Television, Faculty of Applied

Arts, Helwan University

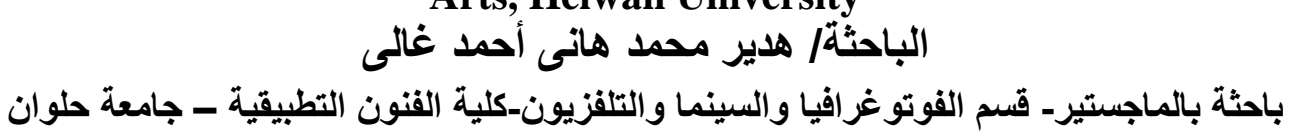

Researcher. Hader Mohamed Hany Ahmed Ghali

Master's Degree - Department of Photography, Film and Television - Faculty of Applied

Arts - Helwan University

aaiconf@aai.edu.eg

لقد اثر التطور التكنولوجي للكاميرات علي شكل المحتوي المرئي فتنقسم كاميرات الفيديو عامه حسب طريقه استخدامها الي Camcorders وكاميرات الميدان Field Cameras وكاميرات الاستوديو Studio Cameras وكاميرات عاليه الحده HDTV Cameras و التى تستخدم غالبا فى انتاج الصوره المتحركه فى الوقت الحالى نظر أ لمميز اتها . وكان ذللك نتيجة للتطور العلمى و التكنولوجى والاكتشافات العلمية والتقدم الصناعى الهائل الذى حدث بالكاميرات من أنو اع و أنكال مختلفة لكلاً منهم الغرض من أستخدامه كان لها الأثر فى تغيير شكل ومفهوم الصورة المتحركة من حيث

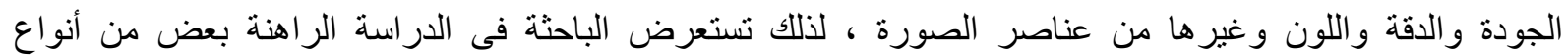
الكاميرات عالية الحدة التى تستخدم حالياً.

\title{
Abstract:
}

Study the Impact of the technological development of the cameras in the production of the Motion Picture and to highlight the language of the image to achieve a distinct image to express the appropriate visual content. study the specifications of the latest types of digital cameras that are currently used and knowledge of the advantages of using digital cameras in the form of the final image resulting in increased clarity and accuracy of the image is less than used the traditional cinema of the price of cameras and printed the film for hundreds of copies and sent back to the cinemas, helped the director to make films easily and control shooting well .

Research problem: The search problem is summarized in:

The difference between the final shape of the motion picture with the technological development of cameras and studying the effect of technology development on visual content.

\section{Research importance:}

- Assisting the specialists in the knowledge of the connection between the technological development of the cameras and the impact on visual content .

- Study the specifications of the latest types of cameras used in the production of motion pictures. 
العدد الثاني عشر - الجزء الثاني

مجلة العمارة والقنون

Research Methodology The researcher follows the descriptive analytical method where the researcher studies the effect of technological development of the cameras on the final form of the motion Picture in the visual content ,The researcher also follows the descriptive method of the latest types of cameras which used to produce the motion picture .

\section{Results :}

Through the study of the impact of technological development of cameras on the final form of the motion picture in the visual content, the researcher concludes the following :

-The difference in image quality resulting from clarity of color and accuracy in image depending on the technological development of each camera type .

- Providing a huge budget for the production companies from printing the film to hundreds of copies and send them to cinemas again in the traditional way in the opposite of digital cinema.

- help the director to make films easily and control shooting well where enabled the director when he finished filming any scene to be aware Show what he had just recorded .

Recommendations : the researcher recommends the following :

- Conducting more analytical studies of the technological development of cameras and the impact of each type on the final form of the image and documenting it with practical applications .

- Developing the curriculum for the specialists with the technological development of the cameras and training them on all available types .

Arri- Amira

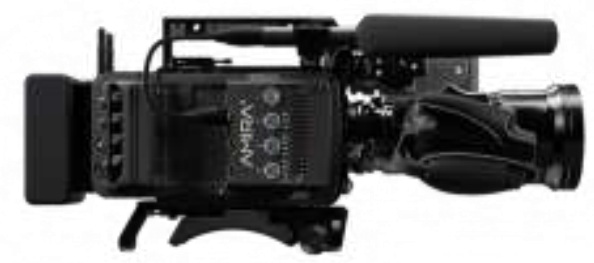

Arri-Amira Aكل(1) لكاميرا

فهى تعتبر الآداه الأمثل للقبام بالتصوير وذلك للمميزات التى توفرها حيث انها تحاكى صوره الـ Alexa حيث تصل بالجوده الى ما يصل 200 اطار فى الثانيه ويحتوى تصميمها المتميز التوازن الفعال والعديد من الدرجات اللونيه التى لهى توفر ها فى الثكل النهائى للصورة المتحركة . لمان

• حيث تصل الجوده بها الى ما يصل لـ 200 اطار في الثانيه فهى تزيد من قيمه الانتاج بتكلفه أقل

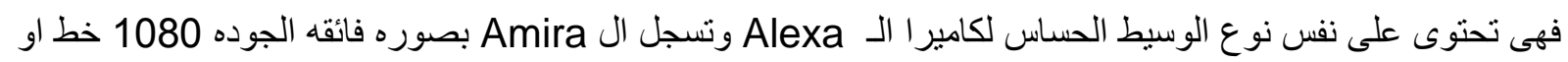
2k التى تعتبر مناسبه للعرض بأى نسبه قياس فى نطاق ديناميكى للسماحيه او التباين قد يصل الى 14 فتحه بمستويات نسبه اضاءه منخفضه مع توفير درجات لونيه للطبيعه و العديد من درجات بشره الجلد فتسعى Amira لتقديم الصور منل دئل 


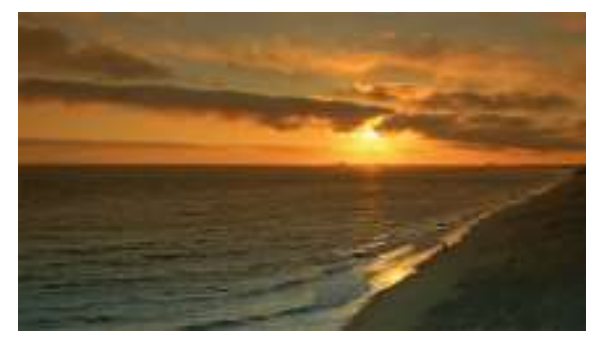

Amira شكل (2) صور طبيعة من كاميرا

• ل العتغرق ال Amira الكثير من الوقت لبدء التشغيل ويمكن استخدامها مباشره من الحقيبه من قبل مستخدم واحد دون تأخير بحيث تكون منوازنه تماما وتوفر الر احه الكامله للمصور فى حين ان محدد الرؤيه الخاص به نوعين من ال

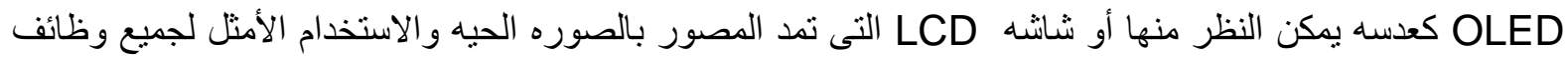
الكامبرا • تعتبر الـ Amira فريده من نوعها حيث انها توفر مرونه غير محدوده فى الصوره والدرجات اللونيه فيمكن ان تستتد لعمل 3D LUT فى تصوير بعض اللقطات اثثاء التصوير بدلا من ذلك انتاج طريقه الـ 3D LUT فى درجات اللون الخارجيه وتحمليها بعد ذلك على الكامير ا اثناء الاعداد وحتى من المكن تعديلها على المشهد اثناء التصوير وايضا يمكن تحميل التعديلات الفرديه على الصوره لمشهد كامل مما يعطى المصوريين و المخرجين درجه أكبر من الابداع على التتاج الصوره المتحركه مع الحفاظ على تكاليف أقل . • على غرار جميع منتجات Arri صمدت الـ Amira لتكون استثمار أمن وطويل الأجل ويتحمل الكثير من الصعاب فى الظروف المحيطه لتحمل مشاق الحياه ، فتعمل تلك الكامير ا بكفاءه عاليه فى اى مكان من صحارى وقمم جبال مغطاه بثلوج و غابات. وقد حصل المخرج الإيطالى Gianfranco Rosi جائزة Golden Bear وهى أعلى جائزة فى مهرجان برلين 2. Amira حيث تم تصوير الفيلم بـ Fire at Sea الدولى على فيلمه التسجيلى التبطى فحينما ظهرت Amira سهلت مهمة تصوير الأفلام النسجيلية حيث يمكن التحكم فى الصوت والصورة بدقة وبسهولة

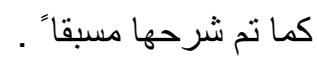

\section{Blackmagic Cinema Camera}

إن السبب فى ظهور الافلام الوثائقيه حاليا بالثكل الأكثر من مدهش هو انه نم تصويرها بكامير ارقميه وبعد ذللك تم تصحيح الالوان بها باستخدام برنامج جيد مثل DaVinci Resolve فكاميرات الفيديو تسجل ولكن بجودة نظهر كالأفلام المنزليه وذلك لانها تقتصر على الوسائط الحساسه العاديه اما Blackmagic فصمدت لكى تحاكى جودة سينمائيه وذلك لمقدرتها على تسجيل نطاق و اسع من التباين وسماحيه التعريض .

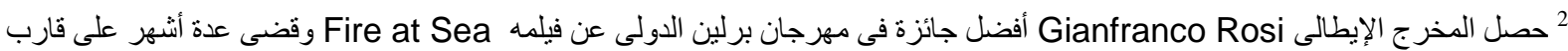

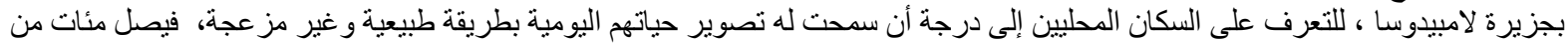

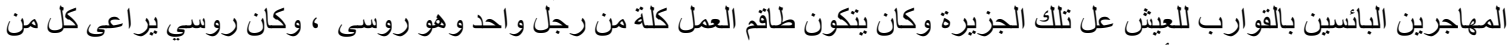

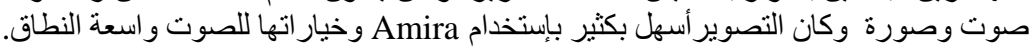




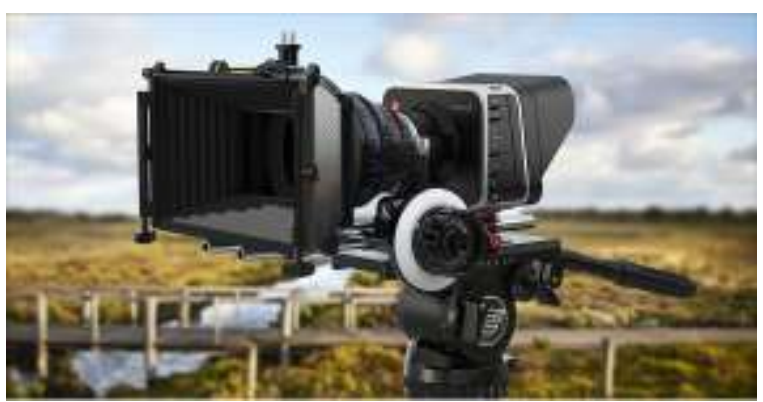

Blackmagic شكل(3) لكاميرا

فإذا كنت بحاجه الى دقه أعلى و عمق ميدانى اعلى وغالق جيد يتحكم بسرعه فال Blackmagic صمدت لذلك الغرض بودة 4k ومساحه وسيط حساس تصل الى 35 مليمتر مزوده بعدسه EF أو فتعتبر هى الخبار الامثل للحصول على صوره عاليه الجودة قد تصل السماحيه فيها الى 12 فتحه عند التصوير وتمكن من التصوير بزاويه واسعه جدا لهديه فنحصل على صوره واضحه وجميله ايضا بنطاق السماحيه والتباين بها قد تمكن من تصوير مناطق الظل والاضاءه العاليه أكثر من اي كامير ا فيديو تقليديه . وصممت الـ Blackmagic بدقه هندسيه عاليه ومن المعدن ذات تشكيل منمق باستخدام احدث الاجهزه الالكترونيه مع امكانيه دعمها بعدسات PL التقليله خلافا لمعظم الكامير ات التى يتكون هيكلها من البلاستيك و وايضا صممت حتى يتمكن

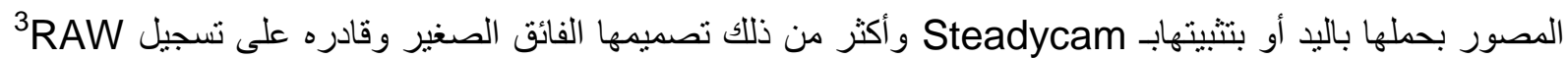

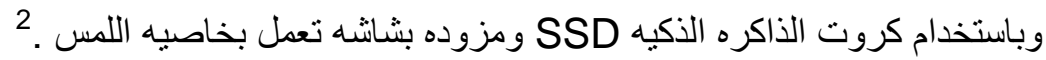
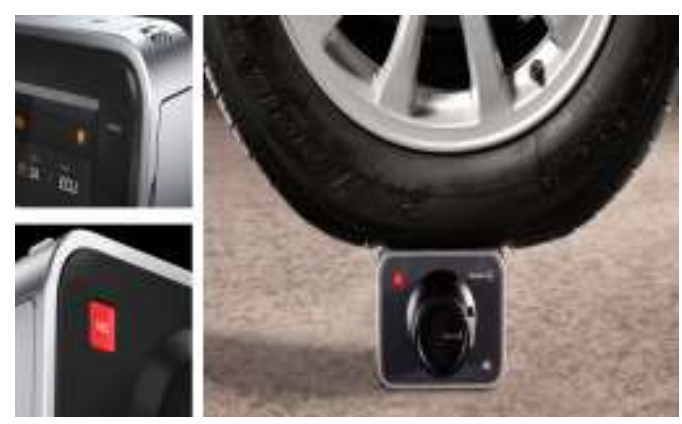

شكل(4) تحمل كاميرا Blackmagic للصدمات والضغط

وبها امكانيه فريده وهى اختيار نوع من نوعين الوسيط الحساس الذى يعمل بجوده 2.5k او 4k ذات نطاق ديناميكى

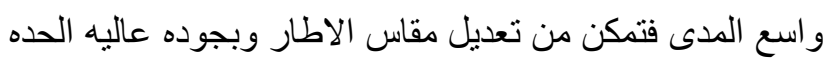
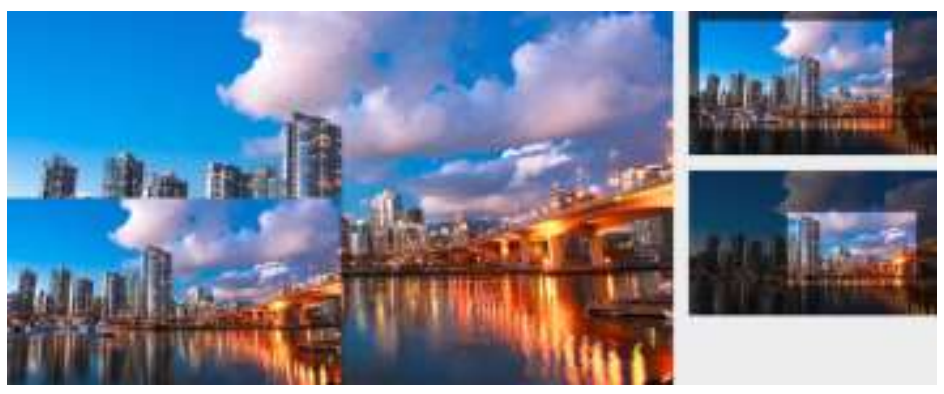

$$
\text { شكل(5) التحكم فى تقسيم الإطار بالكاميرا }
$$

1 Raw file is an image generated by digital cameras, It contains uncompressed, raw image data that can be adjusted for exposure and white balance using software that supports the format. RAW files are used for storing unaltered image data as captured by a digital camera's sensor

${ }^{2}$ https://www.blackmagicdesign.com/products/cinemacameras 
العدد الثاني عشر - الجزء الثاني

مجلة العمارة والقنون

صممت الشاشه المزوده بالكامير ا بإمكانيه العمل باللمس مما تسهل ادخال البيانات على الكامير ا والتحكم مباشر ا فيها حيث يمكن كتابه البيانات على لوحه مفاتيح بها فهى سهله الاستخدام مثل أى هاتف ذكى فهى تمكن المصور من تحديد موقع الملفات بسهوله من داخل الكامير ا والقيام بعمليات ما بعد الانتاج من تصحيح الالوان فمن المكن القيام بعمليات ما بعد

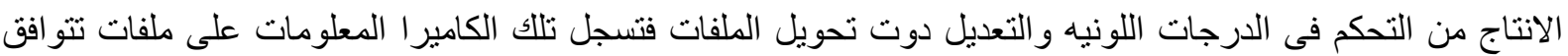
مع جميع التطبيقات الرئيسيه وهذا يعنى انه لا يوجد اهدار للوقت لتحويل البيانات قبل بدء التصحيح اللونى والتعديل فى

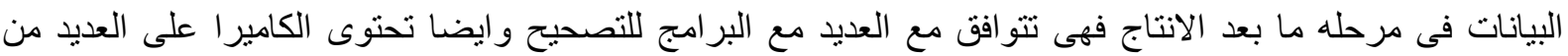
مخارج و أثكال للكابلات مما توفر الكثير من المال امام المستخدم .

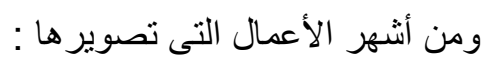

Texting is Dangerous, The One, Hiding Places, Kill Game, Winter in Paris

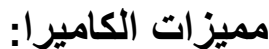

$$
\begin{aligned}
& \text { • يمكن تسجيل البيانات و المعلومات RAW } \\
& \text { • سعر ها مناسب }
\end{aligned}
$$

Arri Alexa

• هى كاميرا رقميه لانتاج الصور المتحركه بجوده عاليه وظهرت فى ابريل 2010 وتعتبر أول انتقال فى صناعه الكاميرات عاليه الجوده بعد Arriflex D-20 و D-21 وتمتاز عدستها بنوع PL وتحنوى على وسيط حساس نوع CMOS مقاس 35 للتصوير بجودة 2880×2160 وتسجل RAW . • ويمكن الحاق الكروت الذكيه بالكامير ا SxS التى يمكن ان تسجل تفاصيل بجوده 709 أو ProRes 422 او ProRes 444 وكذلك 1 مNxHD يمكن تسجيل بجوده تصل الى 1080 خط ، فيمكن أن تصل لالتقاط الى ما يقرب

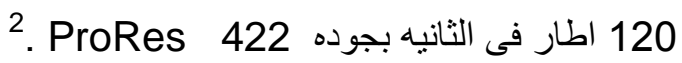
• فصمدت الكامير الانتاج الافلام او البرامج او الاعلانات ذات الميزانيه العاليه فهى تسجل بجوده عاليه على غرار الـ .Thomson Viber g Panavision Gensis g Red 4 يقصد بالأرجنو ميكى او الأرجنو ميكس هو علم يهذف الى تحسين الارتباط بين الانسان و المنتج و البيئة فانة يصبح من الضرورى دراسة كل ما يوثر 2 https://www.arri.com/camera/alexa/ 


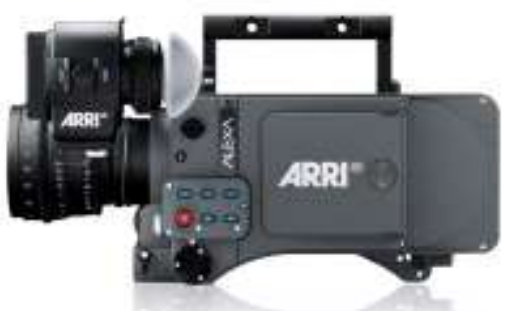

Arri Alexa

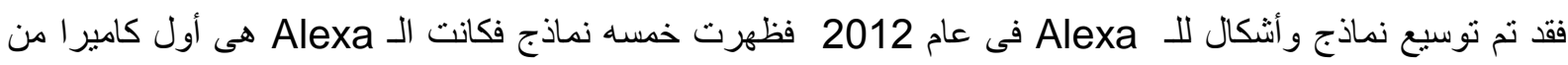
منتجات عائله اليكسا فى ابريل 2010 و ظهر بعدها Alexa Plus و التى تمتاز بامكانيه التحكم عن بعد لاسلكيا ونظام عدسات LDS و ظهر بعدها Alexa Plus 4:3 فهى مناليه لعمل صوره Anamorphic وبعدها Alexa M تمناز

بصغر حجمها وامكانيه التصوير Alexa XT و وبعدها ظهرت Alexa Studio. ومن أثهر الأفلام التى تم تصويرها هى , Brooklyn ,47 Ronin ,Broken City, Macbeth .

RED ONE أعلنت شركة رد لصناعة كاميرات السينما الرقمية أنها سنطرح خلال عام 2009م الكاميرا السينمائية الرقمية "رد وان" Red One. التي ستكون قادرة على تسجيل صور ذات نقاط تصل إلى 4520×2540 باستخدام حساس CMOS وسيكون للكامير ا "رد وان" سعر تنافسي يسمح للإنتاج قليل التكلفة نسبياً بإنتاج سينما رقمية على أحدث تكنولوجيا.

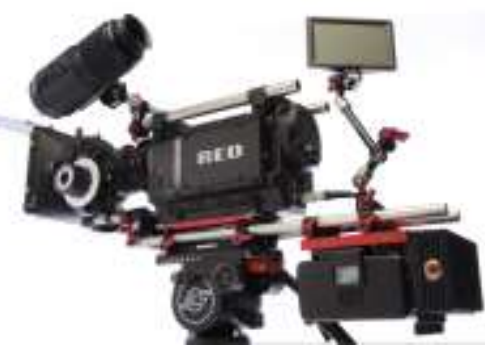

شكل (7) كاميرا

يزن جسم الكاميرا حوالي 8 أرطال وتكون أبعادها: 12" طو لاً × 6.3" ارتفاعًا × 5.2" للعرض. والكرط والكامير ا مصممة على أساس فكرة عامة تعتمد على نظام تركيبي. وله نقاط تركيب عديدة وأجزةة مساعدة مثل أجهزة تسجيل، ومحددات رؤية View Finder و غير ها تركب على الكامبرا بدلاً من أن تكون أجزاء أصلية من الجسموالمحس البصري الخاص بالكاميرا و التي أطلقت عليه اسم ميستريوم

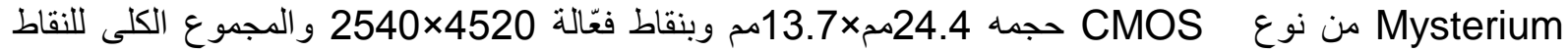
4900×2580 ومجال ديناميكي 66 ديسييل. وحساس الميستريوم له نفس مساحة فعالية إطار الفيلم السوبر 35مم (بنسبة طول إلى عرض 16:9)، مما يسمح بإنتاج نفس عمق المجال الضحل بمصاحبة العدسات المصممة لتغطية نظام

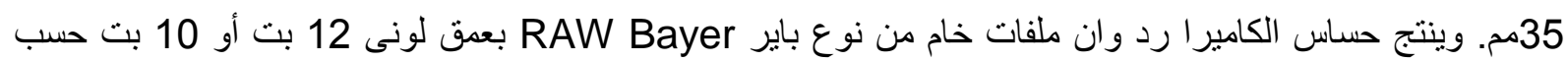
اختيار نوع البيانات الخارجة من الكامير ا. و الكامير انسمح أيضاً باستخدام الحساس في نظام "جزئي" يمكن فيه للحساس أن يحاكى الحيز الفعّال لإطار الفيلم سوبر 16مم و هو يلتقط صورة بجودة 2K وذلك يتيح استخدام الكامير ا بعدسات مصممة لتغطية نظام السوبر 16مم. 
العدد الثاني عشر - الجزء الثاني

مجلة العمارة والقنون

إن نظام Red Code الضاغط الخامى (Raw Codec) سوف يتيح تسجيل بيانات الحساس 4K بمقدار 24 إطار في الثانية بمعدل بيانات حوالي 27.5 ميجابايت/ثانية. و هذا المعدل للبيانات منخفض بما يكفى لكي يصبح من المكن

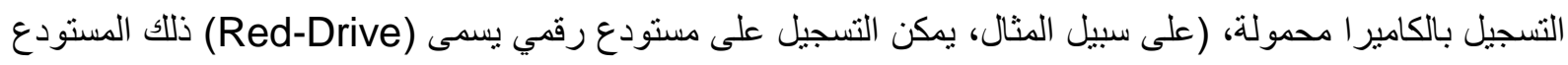
يحتوى على هارد ديسكين 2.5"). وفى النهاية يمكن التمتع بما تحمله تقنية الخام RAW من مميزات، ولكنه يحتوى على

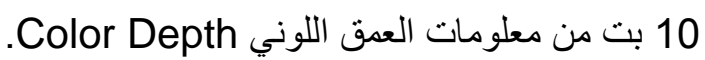

الصورة الخارجة من الكاميرا الصورة التي نم عرضها للكامبرا رد وان والتي تعتمد على المحس البصري الميستريوم 4K في المؤتمر العالمي للإذاعة International Broadcasting Convention, من خلال جهاز عرض 4K ماركة سوني Sony SRX-R110, بدت الصورة شديدة النقاء، ولها مجال ديناميكي مبهر، ونتيجة أدائية جيدة لدرجات البشرة والإضاءة 5 العالية وأستحوذت كامير ا Red على رضاء صانعى الأفلام سواء تسجيلية أو دراما ولكن كثر أس تخدامها فى أفلام الدراما نظر اء لتكلفتها العالية ومن أهم الأعمال التى تم تصوير هاء Independence Day , Room ,Bring Him Home, Hobbit , Tarazan و هناك بعض الأعمال نم تصوير مشاهد بها بـ Red وهى : Avatar2 وفيلم Civil War captain America, Jurassic World و المخطط له العرض في 25 ديسمبر 2017 و أيضا فيلم Guardians of The Galaxy Vol.2 المقرر عرضه 5 مايو 2017.

\section{RED EPIC-W 8K S35 Sensor}

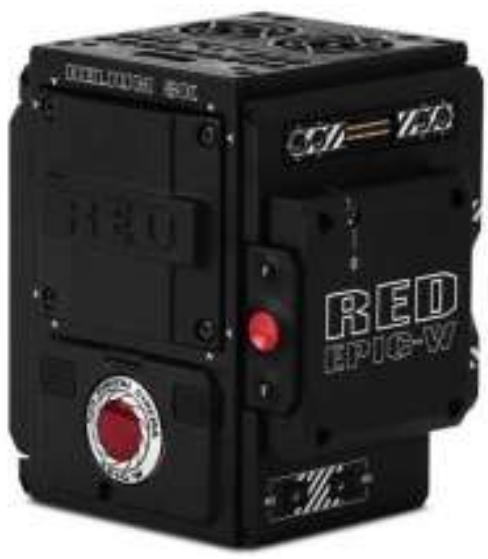

RED EPIC-W 8K شكل (8) كاميرا

تتميز بجودة الصورة الفائقة و أفضل دقة فى فئتها ومزودة بوسيط HELIUM 8K S35 الذى يتيح للمبدعين بتسجيل أدق التفاصيل بالصورة المتحركة قد تصل نسبة الوضوح إلى 35 مليون بكسل لتلنقط كل التفاصيل الصغيرة والدقيقة

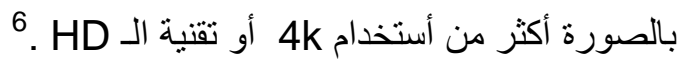

${ }^{5}$ Steven Ascher,Edward Pincus: The Filmmaker's Handbook,A Comprehensive Guide for the Digital Age,2013 Edition,p.120

${ }^{6 \text { Noah }}$ Kadner, RED, The Ultimate Guide to Using The revolutionary Camera , 2010,p.130 


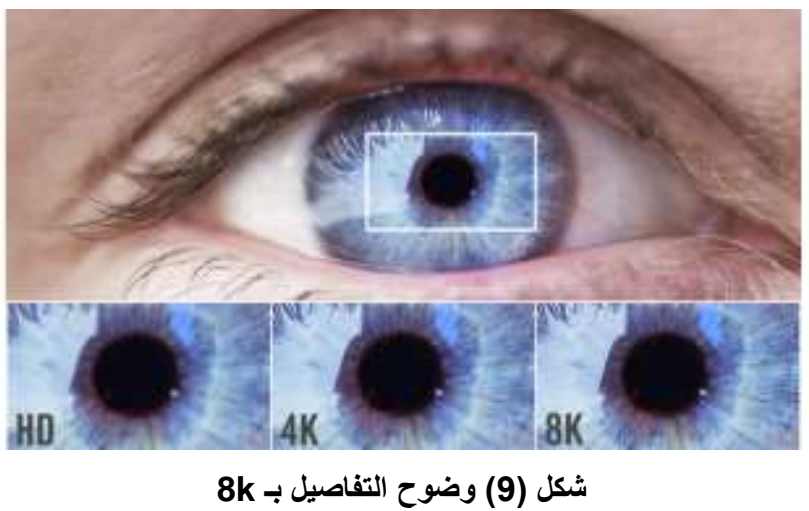

وتقوم بتصوير الألوان بنطاق ديناميكى لا يصدق فتكون النتيجة الحصول على صورة سينمائية متحركة بالألوان الزاهية بإضافة الظلال الموجودة بالصورة للحصول على أكثر صورة واقعية مدكن أن تتخيلها العين البشرية.

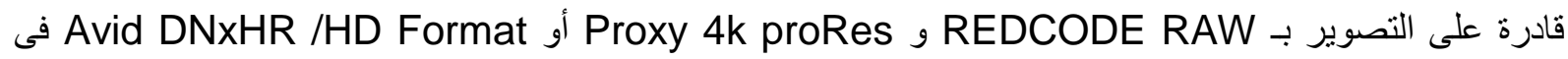
وقت و احد ، ولكن يعيبها زيادة سعر ها .

Arriflex D-20 الكاميرا الأريفليكس السينمائية فائقة الجودة

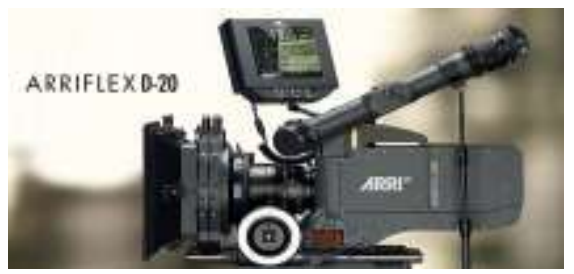

Arriflex D20 شكل (10) كاميرا

تستخدم هذه الكامير ا شر ائح CMOS الحساسة التي تتساوى في الحجم مع حجم الفيلم Super 35m. ونتيجة لذللك فإن الكاميرا D-20 مؤهلة لاستخدام كل العدسات السينمائية المتاحة للكامير ا 35مم التقليدية وبالتالي توفر خصائص بصرية عالية و لا تقلل من فرص الإبداع. 7 تم تصميم الكاميرا بحيث تكون قريبة إلى حد كبير من شكل الكاميرا السينمائية, بالإضافة إلى الاختيارات الكثيرة المتاحة من العدسات السينمائية ومحدد الرؤية البصري واضح الرؤية والملون الذي يوفر صورة عالية الجودة لتحديد الوضوح و التكوين بشكل أكثر سهولة ويسر ومن أهم مميزته أنه يعطي صورة أكبر في المساحة مما تسجلها الثر ائح الحساسة، كما أنه يوفر مساحة أكبر من الصورة التي تسجلها الثريحة الحساسة فيعطي فرصة أكبر لضبط التكوين و الحركة بالإضافة إلى أنه يستخدم طاقة قليلة فبالتالي مناح استخدامه في حالة استخدام البطارية ومر آة غالق دو اره صامته وتتيح هذه الكامير التها القدرة على تسجيل الصور بسر عات عالية والتحكم في السرعة بالإضافة إلى أنها منو افقة مع أغلب الإكسسوار ات ات المتاحة للكامير ات الأريفليكس التقليدية . فقامت تلك الكامير الأريفلكس D-20 طفرة عند دخولها فى مجال تصوير الصورة المتحركة و أثرت فى المحتوى المرئى أيضاء فى الفيلم التسجيلى لما لها من مميزات ومن أشهر الأعمال التى تم أستخدامها فى تصوير ها :

Prisoners of the Sun "2013" , Hogfather "2006" , Captain Abu Raed , Uncertainty"2008" , RocknRolla"2008", Corpse Bride "2005" 
Canon EOS C300 Mark II تعتبر كاميرا Canon EOS C300 Mark II ثورة فى الجيل الثانى لنظام Cinema EOS 4k بنطاق واسع من

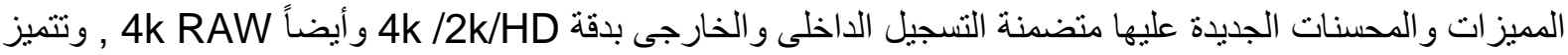
هذة الكاميرا بطاق ديناميكى واسع قد يصل إلى 15 فتحة مزودة بـ Dual Pixel CMOS بنظام جديد من ضبط

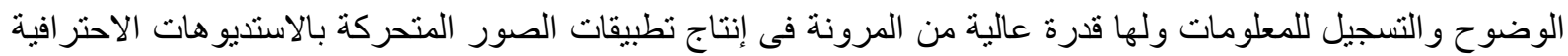

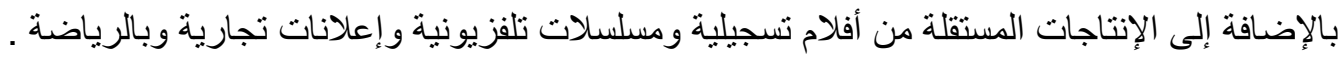

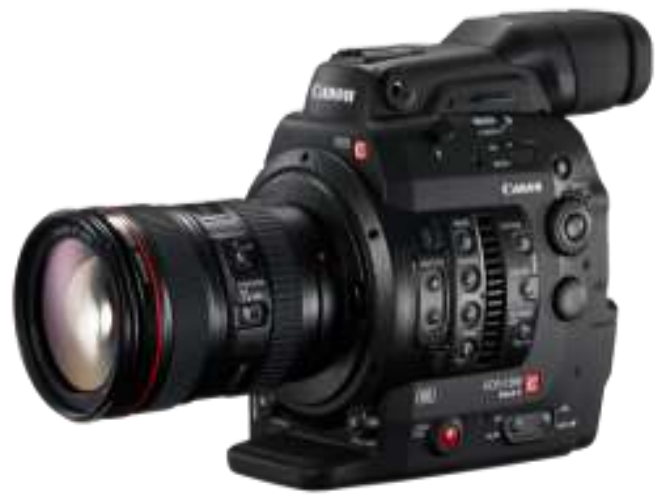

Canon EOS C300 Mark II امكل (11) كاميرا

وبها أيضاً نظام إعادة تصميم للكادر المطلوب تصويره وتحتوى على مستشعر للصور تم تطويره لـ 8.85 ميجابيكسل

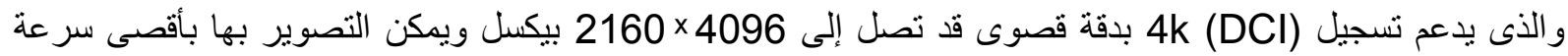
(4) (54 dB) ISO 400 ، لإلتقاط صورة حادة وواضحة بكافة التفاصيل بإستخدام الإضاءة المحيطة فقط وبها مرونة عالية من خلال توافق الكامبرا مع مجموعة كبيرة من العدسات قد تصل إلى أكثر من 100 عدسة بإنتلاف الأنواع .

توفر عدسة EF الثثبتة على EOS C300 تو افقًا ليس فقط مع مجموعة Canon الو اسعة من عدسات DSLR ولكن

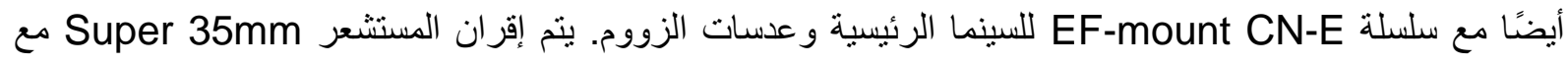

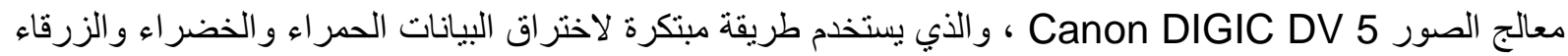
لتمكين أداء الألوان على مستوى يتطلب خلاف ذلك ثلاثة رقائق منفصلة. لمزيد من المرونة لضبط التصوير أثناء مرحلة التباء

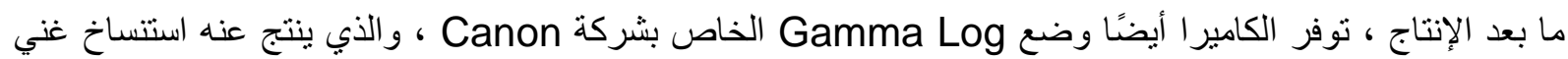
بالدرجات اللونية في مناطق الضوء و الظلال في الصورة بنطاق ديناميكي موسع.

\section{الكاميرا جينيسيس Genesis من شركة Panavision}

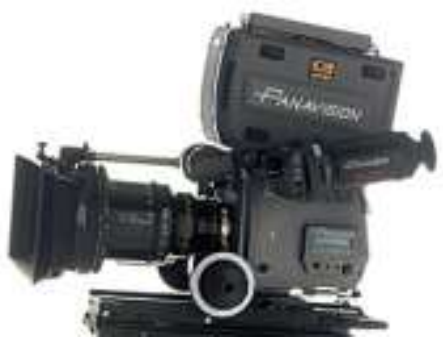

شenesis تشل (12) كاميرا

$1{ }^{\text {Chris }}$ topher Grey, Canon DSLR , Focal press , 2008 ,p.47 
العدد الثاني عشر - الجزء الثاني

مجلة العمارة والفنون

ونتيجة أخرى للتعاون بين شركتي سوني وبانافجن الكامير ا Genesis التي تصل فيها حجم الثريحة الحساسة إلى حجم

مساوي للفيلم سوبر 35مم وبالثالي فهي منو افقة مع كل العدسات السينمائية المستخدمة مع الكاميرات 35مم بالإضافة إلى

Dمق مجال كبير Depth of field ومدى حيوي واسع Daynamic Rang

حجمها ووزنها وتو افقها مع المواصفات الأرجونومية يجعلها ملائمة لكافة الاستخدامات سواء محمولة أو للعمل داخل الأستوديو فهي قابلة للاستخدام على الاستيدي كام أو محمولة أو على كرين أو حتى تستخدم للتصوير تحت الماءونه الماء في 9housing أو مركبة على حوامل التثبيت على السيار ات فهي توفر حرية كبيرة للمصور وقابلة للتثبيت أو الاستخدام تحت أبي ظروف: • ملائمة ومنو افقة مع كل إكسسو ارات البانافن. • سرعة التصوير من 50:1 كادر/ث بالإضافة إلى السرعات الثابتة 23,976 كادر/ث ،24 كادر/ث ،25 كادر/ث ، 29,97 كادر/ث ، 30 كادر/ث.ث. • تعمل بجودة تصل إلى 12.4 ميجابكسل.

• وتنتخدم وصلة ألياف بصرية للتحكم في الكامير التتم عملية تسجيل البيانات بدون ضغط. • • في العادة تكون حساسيتها • يمكن التحكم في زاوية الغالق من 0.8 إلى 360. تحنوي على نظام مدمج للتحكم في الكامير اللتحكم في الوضوح و والزوورم و فتحة العدسة. 10 وتم أستخدامها مؤخر اء فى كثير من الأعمال التسجيلية والدرامية حيث كان لها دور فعال على المحتوى المرئى للصورة المتحركة نظر اء لحساسيتها العالية التى تمكن مدير التصوير من التصوير فى أماكن الإضاءة الضعيفة مع جودة عالية للصورة ومن أهم تلك الأعمال :

Drop Dead Diva "2014", Lost Girl " 2010" , Breakout Kings "2012",

CSI: Miami "2012" „Captain America : The First Avenger"2011" , Parental Guidance"2012" ,Californication"2014" ,2012 "2009", Heaven Is for Real "2014"

Crittercam

و أنتجت ناشيونال جيوجر افيك الكريتركام Crittercam وهى عباره عن كامير ا صممت خصصيا لتتبت على الحيو انات حتى تعطى صوره حيه دوت تدخل الانسان و حتى تعكس صوره الحياه البريه طبيعيه فظهرت اولا للحيوانات البحريه وكانت تتبت عن طريق و عاء خاص و فقا للجو المحيط بالحيو ان , وبعد ذلك ظهرت للحيو انات البريه .

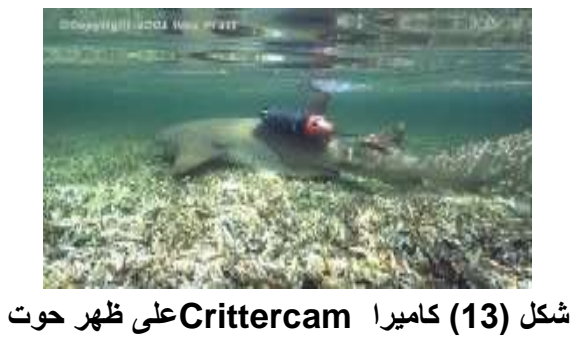

9 و هو عبارة عن حافظة من الزجاج توضع بداخلها الكامير اللتصوير تحت الماء

${ }^{10}$ Gerald Millerson ,Jim Owens:Video Production Handbook, Fourth Edition ,focal press,p.80 2 http://animals.nationalgeographic.com/animals/crittercam/ 
و اختلفت شكل الكريتركام على مدار السنين مع التطور التكنولوجى حتى ظهرت اليوم بالثكل الصغير ولتحملها العديد من الظروف الصعبه المحيطه بالحيوان.

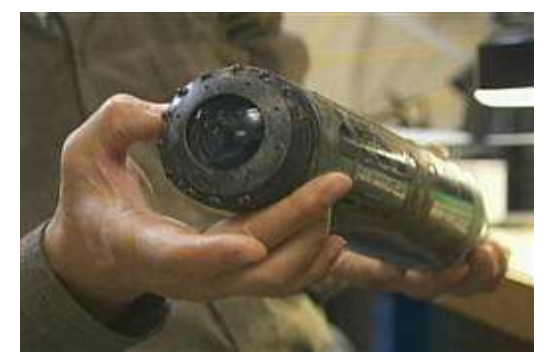

\section{شكل (14) أخر شكل لا Crittercam}

فقد طر أت العديد من التغيير ات على الكريتركام منذ اختر اعها من تصغير للحجم والوزن لتصل حاليا لكيلو و الذى لا يمثل جزء كبير من وزن الحيوان المثبته به بحيث تتناسب معه وتتحمل الظروف المحيطه به. ونجحت ناثيونال جيوجر افيك لاصدار كريتركام تعمل بالكروت الذكيه بدلا من شرائط الفيديو التى تؤثر على التصوير بهرئ

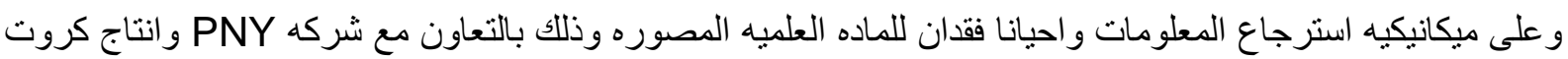

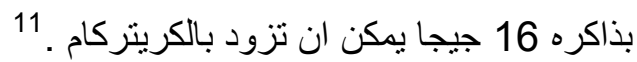
أما الكريتركام التى تستخدم مع حيوانات اليابسه أكثر تطورا بحيث تتيح فريق العمل بنقل الصوره مباشرأ عن طريق موجات وتتيح لهم التحكم فى بدء والانتهاء من التصوير ويمكن ايضا تحديد موقع الكريتركام عن طريق GBS مزوده به حيث يمكن تحرير الكريتركام من الحيوان حال تسببها بضرر له ه.

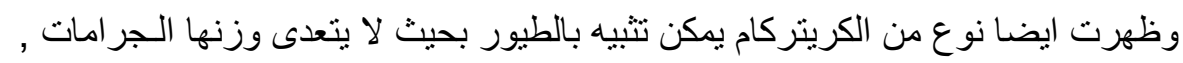

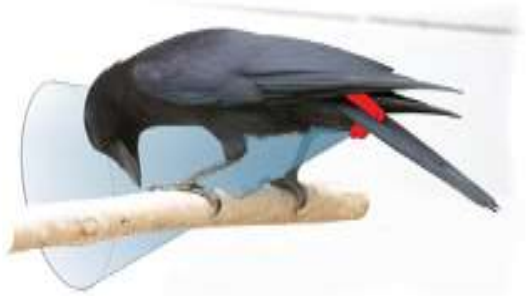

شكل (15) كامير ا كريتركام بالطير

من خلال الدراسة لموضوع نأثثير التطور التكنولوجى للكاميرات على الثنكل النهائى للصورة المتحركة فى المحتوى

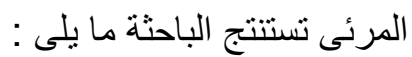
- اختلاف جودة الصورة الناتجة من وضوح اللون و الدقة فى وضوح الصورة تبعاً للنطور التكنولوجى لكل نوع كامير ا. - توفير الميز انية الضخمة على شركات الإنتاج من طبع الفيلم لمئات النسخ وإرسالها لدور العرض مرة اخرى بالطريقة التقليدية عن السينما الرقمية التى وفرت الكثير بفضل الكاميرات الرقمية الحديثة . - زيادة وضوح ودقة الصورة النهائية بالكامبرات الرقمية الحديثة فى نفس ظروف الإضـاءة المحيطة بالششه دون التكلف فى مصادر الإضـاءة . 
- السهولة في خلق وصنع الفيلم والتحكم بلقطاته , ووجود اختصار ات للعديد من العمليات المرهقة عن النظام التقليدي,

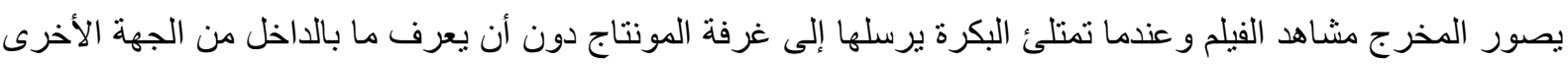
فالكامير الرقمية تئمن المخرج عند انتهائه من تصوير أي مشهد أن يعيد عرض ما قام بتسجيله للتو وهذا مريح جداً

- - عمل المزيد من الدراسات التحليلية للتطور التكنولوجى الحديث للكاميرات وتأثثير كل نوع على الثكل النهائى للصورة ونوثيق ذلك بتطبيقات عملية .

- تطوير المناهج الدراسية للمتخصصين فى التصوير بالتطور التكنولوجى للكاميرات وتدرييهم على كل الأنواع المتاحة وذللك إسهاماً فى تكوين هوية المصور الجيدة .

1- ${ }^{\text {Chris }}$ topher Grey, Canon DSLR, Focal press , 2008.

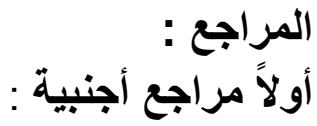

2- Gerald Millerson ,Jim Owens:Video Production Handbook, Fourth Edition ,focal press.

3- Jon Fauer, Arriflex 35 Book, Third Edition, Focal Press

4- ${ }^{\text {Noah }}$ Kadner, RED, The Ultimate Guide to Using The revolutionary Camera, 2010 .

5- Steven Ascher,Edward Pincus: The Filmmaker's Handbook,A Comprehensive Guide for the Digital Age,2013 Edition .

6- http://www.arri.com

7- https://www.blackmagicdesign.com/products/cinemacameras

8-https://www.arri.com/camera/alexa/

9- https://en.wikipedia.org/wiki/Arriflex_D-20

10- http://animals.nationalgeographic.com/animals/crittercam/ 